\title{
Emergency Department Utilisation Among Older Adults - Protocol for a Systematic Review of Determinants and Conceptual Frameworks
}

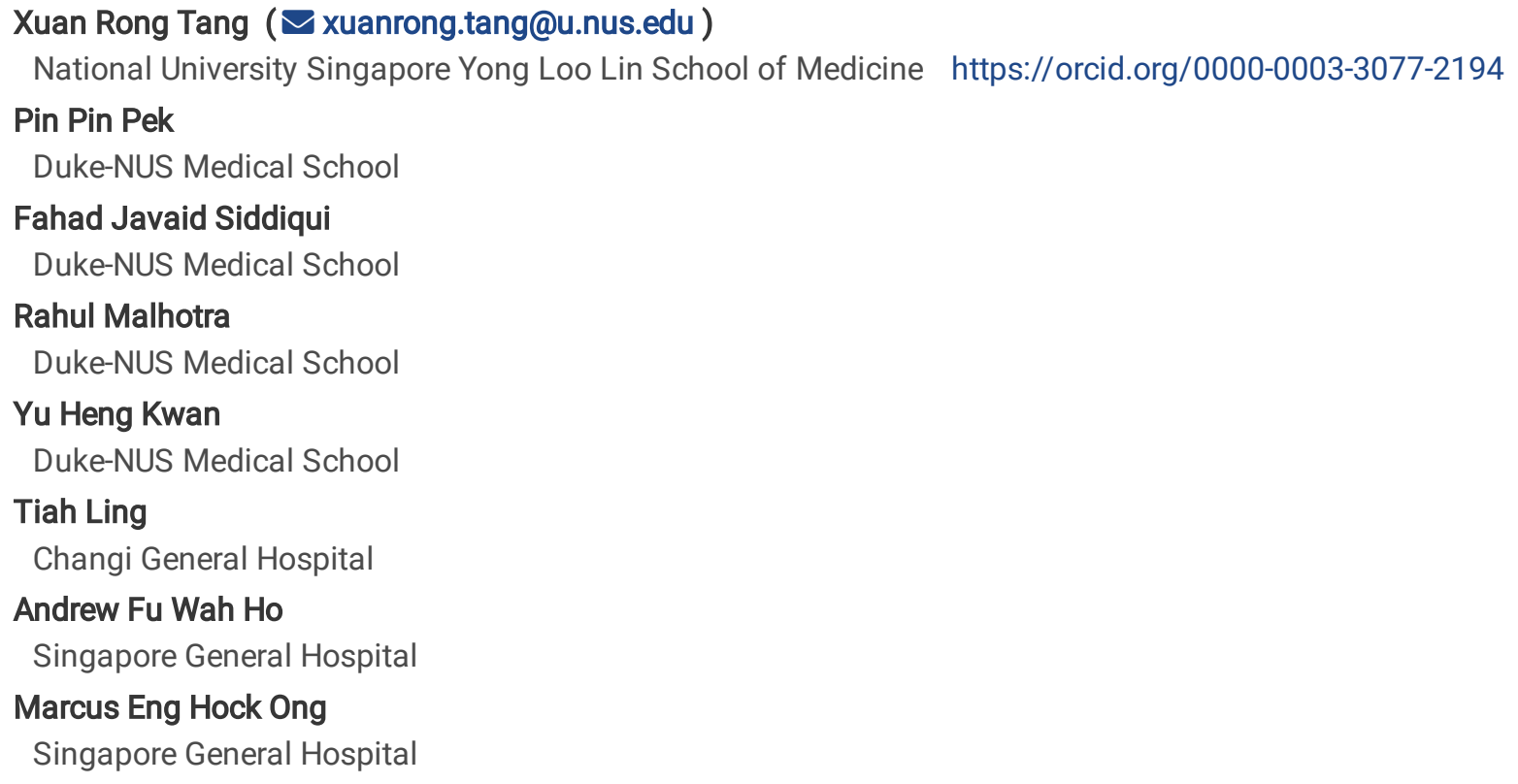

Protocol

Keywords: aged, geriatric, elderly, aging, ageing, health services, emergency department, emergency medical services, emergency service hospital, healthcare utilisation

Posted Date: May 18th, 2021

DOI: https://doi.org/10.21203/rs.3.rs-508927/v1

License: @ (i) This work is licensed under a Creative Commons Attribution 4.0 International License. Read Full License 


\section{Abstract}

Background

Older adults aged 65 years and above have a disproportionately higher utilization of emergency healthcare, of which Emergency Department (ED) visits are a key component. They tend to require more extensive workup, therefore spending a greater amount of time in the ED. The rise in the older adult population globally may contribute to worsening overcrowding in many EDs. When older adults visit the ED, they are at higher risk of hospitalisation and adverse events compared to younger patients. Following discharge from the ED, older adults often experience functional decline and reduction in mobility, which may not improve within a year. In this paper, we present a protocol for a systematic review of the determinants of ED utilisation among community-dwelling older adults aged 65 years and above, applying Andersen and Newman's model of healthcare utilisation. Furthermore, we aim to present other conceptual frameworks for healthcare utilisation and propose a holistic approach for understanding the determinants of ED utilisation by older persons.

\section{Methods}

The protocol is developed in accordance with the standards of Campbell Collaboration guidelines for systematic reviews, with reference to the Cochrane Handbook for Systematic Review of Interventions. Medline, Embase and Scopus will be searched for studies published from 2000 to 2020. Studies evaluating more than one determinant for ED utilisation among older adults aged 65 years and above will be included. Search process and selection of studies will be presented in a PRISMA flow chart. Statistically significant $(p<0.05)$ determinants of ED utilisation will be grouped according to individual and societal determinants. Quality of the studies will be assessed using Newcastle Ottawa Scale (NOS).

\section{Discussion}

In Andersen and Newman's model, individual determinants include predisposing factors, enabling and illness factors, and societal determinants include technology and social norms. Additional conceptual frameworks for healthcare utilisation include Health Belief Model, Social Determinants of Health and Big Five personality traits. By incorporating the concepts of these models, we hope to develop a holistic approach of conceptualizing the factors that influence ED utilisation among older people.

Systematic review registration

This protocol is registered on 8 May 2021 with PROSPERO's International Prospective Register of Systematic Reviews but pending confirmation.

\section{Background}

The emergency department (ED) acts as a bridge between the community and hospital, where people are referred by their primary care physicians (PCPS) or by themselves, and plays a crucial role in regulating hospital admissions (1). In the healthcare system, the ED is vital in supporting primary care by caring for patients outside of office hours or performing advanced diagnostic investigations (1). In the recent years, there has been a surge of ED visits $(2,3)$, as seen in the United States where the number of visits per 1000 people have increased from 369 to 458 visits between 1995 to 2016 (4). Similarly in Singapore, the number of ED visits have risen by 250,000 from 2007 to 2013 (5). Adults aged 75 years and above had the second highest visit rate, of 52 visits per 100 people, after infants younger than one year old (6). The number of older persons, aged 65 years and above, is expected to rise (7), so is the frequency of ED utilisation by this population sub-group (4).

Older adults contribute a disproportionate number of visits to the $\operatorname{ED}(8,9)$ and tend to require more extensive workup, therefore spending a greater amount of time in the ED (4). Furthermore, there is higher resource expenditure among this population in the form of advanced investigations such as computed tomography and magnetic resonance imaging (2). Compared to their younger counterparts, they are at higher risk of hospitalisation as well as adverse events when they visit the ED (10). In the United States, expenses incurred from inpatient care accounts for $31 \%$ of national healthcare spending (1). In addition, they have an increased susceptibility to hospital acquired pneumonia (11). Older adults who were discharged from the ED had a reduction in their mobility within the community, which may not improve within a year from discharge (12). 
Generally, older adults have multiple comorbidities and complex medical issues that may require care beyond the PCP level (5). In a systematic review done by McCusker (2003) adapting Andersen's Behavioural model to study the determinants of ED utilisation (13), need or illness factors were shown to be a significant determinant across many studies. This signifies that older adults truly require emergent care and may be too acutely ill to await an appointment at the PCP. In certain situations, the process of deterioration could have been deterred with regular follow-up care with PCPs (14). Moreover, McCusker found that predisposing and enabling factors that increase use of PCP will lead to a decrease in ED utilisation (13). Indeed, the presence of barriers to primary care was identified as one of the reasons why older adults turn to the ED in desperation to resolve their issues. In fact, some were told by the PCP staff to visit the ED if they felt it was urgent (15). Even though the illness factor was the predominant individual-level determinant in McCusker's study, there exists a multitude of factors that influence the utilisation of ED that should be discussed as well. This is visualised through the model proposed by Andersen and Newman (Fig. 1), where healthcare utilisation is determined by societal or individual factors.

Individual determinants include predisposing, enabling and illness factors. Predisposing factors are patient sociodemographic characteristics that can incline or deter a patient from utilising healthcare. Enabling factors encompass the influence of family and community, with examples including marital status, living conditions and geographical accessibility to PCPs or EDs. Need or illness factors can be divided into perceived (subjective) need or evaluated (objective) need $(13,16)$. Societal determinants include technology and norms. Technology will help promote the efficacy of physicians providing care within the healthcare system, which can influence the decision of the population to seek medical care (17). An example of this would be the availability of X-rays and blood investigations at the ED which may not be available at the PCP level (18). Societal norms arise from governmental policies as well as societal values and beliefs (17). For instance, the stigma associated with mental health issues impedes help-seeking behaviour among people who need them and potentially deters them from utilising healthcare (19). Health insurance policies and medical subsidies by the government play a key role in a person's decision to utilise healthcare resources (17), as demonstrated in Anderson's study where healthcare utilisation was lower among people without insurance coverage (20).

The stress on the ED needs to be addressed to avoid jeopardising the quality of care provided and slow the surge in healthcare expenditure (3). The ill effects on older adults outlined above with regards to ED visitation emphasises the need to investigate the determinants of ED utilisation. With identification of these factors, we may be able to mitigate the number of visits to the ED by the older adult population through primary, secondary and tertiary prevention. Hence, in this paper, we outline the protocol for a systematic review of the determinants of ED utilisation among older persons (aged 65 years and above), using the framework proposed by Andersen and Newman as shown in Fig. 1.

In addition to Andersen and Newman's model, other frameworks have been used to explain health services utilization. These include the Health Belief Model $(21,22)$, Social Determinants of Health (23) as well as Big Five personality traits (24, 25). Lutz 2018 devised a framework to understand ED utilisation by describing the factors that influence the decision of visiting the ED or primary care (26). In addition, He et. al proposed a modified Andersen and Newman's model to visualise ED utilisation (27). By incorporating concepts of all the models utilised in our review, we hope to develop a more holistic approach of conceptualizing the factors that influence the decision of older adults to visit the ED.

Our protocol was developed in accordance with the standards of Campbell Collaboration guidelines for systematic reviews (28), with reference to the Cochrane Handbook for Systematic Review of Interventions (29). This protocol is registered with PROSPERO's International Prospective Register of Systematic Reviews but pending confirmation.

\section{Methods}

\section{Objectives}

The primary aim of our study is to conduct a systematic review to understand determinants of ED utilisation among communitydwelling older persons (aged 65 years and above), using the framework proposed by Andersen and Newman as shown in Fig. 1.

The secondary aim is to present other conceptual frameworks that have been used for explaining healthcare utilisation, and develop a more holistic approach of conceptualizing the factors that influence the decision of older persons to visit the ED.

\section{Electronic searches}

Page $3 / 10$ 
Medline, Embase and Scopus will be searched. Additional papers would be identified through handsearching. Grey literature will be searched in OpenGrey. Search will be limited from 2000 to 2020 and papers in the English language only.

The search strategy was developed in collaboration with a university librarian. Medical Subject Headings (MeSH) terms that will be used are "aged", "health services for the aged", "health services accessibility", "health care surveys", "emergency service, hospital" and "emergency medicine". Emtree subject headings that will be used are "aged", "hospital", "emergency health service", "emergency medicine", "emergency ward". Full list of keywords with boolean terms used are available in the appendix.

\section{Inclusion criteria}

Studies that evaluate one or more determinants for ED utilisation among community-dwelling older persons will be included. Use of emergency medical services must be measured in the studies. Papers that are published in English language between 2000 to 2020 will be considered.

\section{Exclusion criteria}

Studies that evaluate determinants for healthcare utilisation in other contexts such as urgent care centres or primary care clinics that are open beyond office hours will be excluded. Papers that merely study presenting complaints of older patients, or studies evaluating determinants of revisits or frequent visits will be excluded.

\section{Selection of studies}

The search and selection process will be displayed in a Preferred Reporting Items for Systematic Review and Meta-analysis (PRISMA) flow chart (30), as shown in Fig. 3.

At least two independent reviewers will conduct the search and selection of studies for each of the databases. A similar search strategy will be applied to each database and citations exported to EndNote X9. Papers identified from references and grey literature will also be included. Duplicates will be removed. Title and abstracts of the search results will then be screened independently for eligibility by two reviewers. Upon screening and shortlisting eligible papers based on the title and abstract, the full text of these articles would be obtained and assessed further for suitability to include in the systematic review. Articles that are excluded for various reasons will be taken note of, in which the justification will be presented in the flow chart and in the manuscript. In the event of any conflict between two reviewers, the opinion of a third, senior reviewer would be consulted.

\section{Data extraction and management}

Training will be provided to reviewers through the Campbell Collaboration website training page, which comprises resources on conducting a systematic review (31). At least two reviewers will be required for data extraction for each article and any discrepancies resolved through discussion and consensus with a third reviewer. The following study characteristics will be identified and extracted from the eligible studies: (1) Author and year, (2) country, (3) study design, (4) study population, (5) sample size and sampling methods, (6) outcome variable(s), (7) data source for outcome(s), (8) determinants examined, (9) data source for determinants. In this table (Table 1), the determinants examined will be classified according to societal determinants (technology and norms) and individual determinants (predisposing, enabling and illness factors). The statistically significant determinants of ED utilisation identified in the studies, together with its effect size, will be presented in the next table (Table 2).

Table 1

Characteristics of included studies (dummy)

\begin{tabular}{|c|c|c|c|c|c|c|c|c|c|}
\hline \multirow{2}{*}{$\begin{array}{l}\text { Author } \\
\text { (year) }\end{array}$} & \multirow[t]{2}{*}{ Country } & \multirow{2}{*}{$\begin{array}{l}\text { Design } \\
\text { of } \\
\text { study }\end{array}$} & \multirow{2}{*}{$\begin{array}{l}\text { Study } \\
\text { population }\end{array}$} & \multirow{2}{*}{$\begin{array}{l}\text { Sample } \\
\text { size and } \\
\text { sampling } \\
\text { methods }\end{array}$} & \multirow{2}{*}{$\begin{array}{l}\text { Outcome } \\
\text { variable }\end{array}$} & \multirow{2}{*}{$\begin{array}{l}\text { Data } \\
\text { source } \\
\text { for } \\
\text { outcome }\end{array}$} & \multicolumn{2}{|c|}{ Determinants examined } & \multirow{2}{*}{$\begin{array}{l}\text { Data source } \\
\text { for } \\
\text { determinants }\end{array}$} \\
\hline & & & & & & & $\begin{array}{l}\text { Individual } \\
\text { determinants }\end{array}$ & $\begin{array}{l}\text { Societal } \\
\text { determinants }\end{array}$ & \\
\hline
\end{tabular}

\section{Data analysis}

The study design and data sources of each study will be presented under the characteristics of included studies in Table 1, together with the list of determinants examined by the various studies. This will provide an overview of the determinants of ED utilisation among patients aged $\geq 65$ years. 
The determinants that are significantly associated with ED utilisation will be identified, through either univariable or multivariable analysis conducted in the included studies. Statistically significant determinants of ED utilisation $(p<0.05)$ will be identified and presented in Table 2. The effect size of the statistically significant determinants will be pooled, if possible, for meta-analysis. Qualitative data will be included for systematic review. Adopting the model in Fig. 1, the determinants will be classified according to individual-level determinants (and therein as illness level, predisposing factors and enabling factors) and societal-level determinants (and therein technology and norms). Determinants that do not fall under this classification will be listed under "other significant factors" - their identification will be useful in informing and proposing modifications to Andersen and Newman's model. If deemed appropriate, we will then devise a modification of the model that will be applicable in the context of ED utilisation.

Table 2

Statistically significant determinants of ED utilisation based on multivariable analysis (dummy table)

\begin{tabular}{|llllll}
\hline $\begin{array}{l}\text { Author } \\
\text { (year) }\end{array}$ & \multicolumn{2}{l}{ Individual determinants } & \multicolumn{2}{l}{ Societal determinants } & $\begin{array}{l}\text { Other significant } \\
\text { factors \& effect size }\end{array}$ \\
\cline { 2 - 5 } & $\begin{array}{l}\text { Predisposing factors } \\
\text { and effect size }\end{array}$ & $\begin{array}{l}\text { Enabling factors } \\
\text { and effect size }\end{array}$ & $\begin{array}{l}\text { Illness level } \\
\text { and effect size }\end{array}$ & $\begin{array}{l}\text { Technology } \\
\text { and effect size }\end{array}$ & $\begin{array}{l}\text { Norms and } \\
\text { effect size }\end{array}$
\end{tabular}

\section{Criteria for assessing the quality of the qualitative evidence}

The Newcastle Ottawa Scale (NOS) will be used for evaluation of the quality of included studies, which will comprise mainly nonrandomised studies (32). The criteria for evaluation in NOS and reliability between raters have been validated (33). As the original NOS only includes assessment of quality of cohort and case-control studies, a modified version of the scale would be adopted for cross-sectional studies. This modified NOS has been used by a prior study (34) with similar criteria of assessment - selection, comparability and outcomes of study. A copy of both original and modified NOS are included in the appendix.

Table 3

Quality assessment of included studies using NOS (dummy table)

\section{Dealing with missing data}

In the event of missing information (such as effect sizes of statistically significant determinants), the corresponding authors of the relevant studies would be contacted to obtain the missing data.

\section{Discussion}

Using Andersen and Newman's model of healthcare utilisation, there is a comprehensive viewpoint on the determinants of ED utilisation which takes into account geographical distance and variations in healthcare systems across different societies. According to the World Health Organisation, older persons are aged 65 years and above, hence justifying our cut-off age (35). Even though our review includes only older patients aged 65 and above, some of the determinants (such as geographical distance) could also be applicable to younger age groups as they are not exclusive to the older adult population. Therefore, our study findings have the potential to be generalised across other populations.

One limitation of our study is the exclusion of non-English papers, which could limit the generalisability of our findings to non-English speaking countries.

Ethics approval is not required as this is a systematic review. We intend to submit the completed review for peer-reviewed publication and to present our findings at relevant meetings and conferences.

\section{Preliminary timeframe}

The table below presents the estimated duration required for each stage of the systematic review. In total, the review would take an estimated 4 months to complete. 


\begin{tabular}{|ll|}
\hline Stage of review & Duration (weeks) \\
\hline Training and pilot testing on the inclusion criteria & 3 \\
\hline Search for eligible results & 2 \\
\hline Screening results from the literature search & 2 \\
\hline Training and pilot testing the study coding procedure & 2 \\
\hline Extraction and analysis of data from eligible research reports & 3 \\
\hline Preparation of the final review report & 4 \\
\hline
\end{tabular}

\section{Plans for updating the review}

No plans are made to update the systematic review at the time of writing. In the event of any changes to the original protocol, they will be presented in the published review.

\section{Declarations}

\section{Ethics approval and consent to participate}

Ethical approval was not required as all data used was derived from aggregated public sources and contained no individually identifying information.

\section{Consent for publication}

All authors give consent for publication.

\section{Availability of data and materials}

Data is available upon reasonable request.

\section{Competing interests}

There are no competing interests to declare.

\section{Funding}

AFWH was supported by the Estate of Tan Sri Khoo Teck Puat (Khoo Clinical Scholars Programme), Khoo Pilot Award (KP/2019/0034), Duke-NUS Medical School and National Medical Research Council (NMRC/CS_Seedfd/012/2018).

MEH Ong reports grants from the Laerdal Foundation, Laerdal Medical, and Ramsey Social Justice Foundation for funding of the Pan-Asian Resuscitation Outcomes Study. This study is supported by grants from the National Medical Research Council (Singapore), Ministry of Health, Singapore.

\section{Authors' contributions}

AFWH conceived of the study. All authors contributed to the manuscript.

\section{Acknowledgements}

We are grateful for Ms Wong Suei Nee for her valuable contribution in developing the search strategy for our systematic review protocol.

\section{Appendices}

Newcastle Ottawa Scale (case-control and cohort)

Modified Newcastle Ottawa Scale for cross-sectional studies 
Full search strategy with concepts and keywords for each database used

\section{References}

1. Morganti KG, Bauhoff S, Blanchard JC, Abir M, lyer N, Smith A, et al. The Evolving Role of Emergency Departments in the United States. Rand Health Q [Internet]. 2013 Jun 1 [cited 2021 Apr 26];3(2). Available from: https://www.ncbi.nlm.nih.gov/pmc/articles/PMC4945168/

2. Pines JM, Mullins PM, Cooper JK, Feng LB, Roth KE. National Trends in Emergency Department Use, Care Patterns, and Quality of Care of Older Adults in the United States. J Am Geriatr Soc. 2013;61(1):12-7.

3. O'Malley AS, Gerland AM, Pham HH, Berenson RA. Rising pressure: hospital emergency departments as barometers of the health care system. Issue Brief Cent Stud Health Syst Change. 2005 Nov;(101):1-4.

4. The Latest Emergency Department Utilization Numbers Are In [Internet]. ACEP Now. [cited 2021 Apr 26]. Available from: https://www.acepnow.com/article/the-latest-emergency-department-utilization-numbers-are-in/

5. Boh C, Li H, Finkelstein E, Haaland B, Xin X, Yap S, et al. Factors Contributing to Inappropriate Visits of Frequent Attenders and Their Economic Effects at an Emergency Department in Singapore. Acad Emerg Med. 2015;22(9):1025-33.

6. Products - Data Briefs - Number 401 - March 2021 [Internet]. 2021 [cited 2021 Apr 26]. Available from: https://www.cdc.gov/nchs/products/databriefs/db401.htm

7. Garson A, Levin SA. Ten 10-Year Trends for the Future of Healthcare: Implications for Academic Health Centers. Ochsner J. 2001 Jan;3(1):10-5.

8. Gruneir A, Silver MJ, Rochon PA. Review: Emergency Department Use by Older Adults: A Literature Review on Trends, Appropriateness, and Consequences of Unmet Health Care Needs. Med Care Res Rev. 2011 Apr 1;68(2):131-55.

9. Ukkonen M, Jämsen E, Zeitlin R, Pauniaho S-L. Emergency department visits in older patients: a population-based survey. BMC Emerg Med. 2019 Feb 27;19(1):20.

10. Aminzadeh F, Dalziel WB. Older adults in the emergency department: A systematic review of patterns of use, adverse outcomes, and effectiveness of interventions. Ann Emerg Med. 2002 Mar 1;39(3):238-47.

11. Min J, Kim H-J, Yoon C, Lee K, Yeo M, Min K. Hospital-Acquired Pneumonia among Inpatients via the Emergency Department: A Propensity-Score Matched Analysis. Int J Environ Res Public Health. 2018 Jun;15(6):1178.

12. Brown CJ, Kennedy RE, Lo AX, Williams CP, Sawyer P. Impact of Emergency Department Visits and Hospitalization on Mobility Among Community-Dwelling Older Adults. Am J Med. 2016 Oct;129(10):1124.e9-1124.e15.

13. McCusker J, Karp I, Cardin S, Durand P, Morin J. Determinants of emergency department visits by older adults: a systematic review. Acad Emerg Med Off J Soc Acad Emerg Med. 2003 Dec;10(12):1362-70.

14. Derlet RW, Richards JR. Ten Solutions for Emergency Department Crowding. West J Emerg Med. 2008 Jan;9(1):24-7.

15. D'Avolio DA, Strumpf NE, Feldman J, Mitchell P, Rebholz CM. Barriers to Primary Care: Perceptions of Older Adults Utilizing the ED for Nonurgent Visits. Clin Nurs Res. 2013 Nov 1;22(4):416-31.

16. Aday LA, Eichorn RL. The Utilization of Health Services: Indices and Correlates: A Research Bibliography, 1972. National Center for Health Services Research and Development; 1972. 126 p.

17. Andersen R, Newman JF. Societal and Individual Determinants of Medical Care Utilization in the United States. Milbank Q [Internet]. 2005 Dec [cited 2021 Apr 8];83(4). Available from: https://www.ncbi.nlm.nih.gov/pmc/articles/PMC2690261/

18. Bornais JAK, Crawley J, El-Masri MM. One Stop: Examining the Reasons Patients Use the Emergency Department for Nonurgent Care and the Barriers They Face. J Emerg Nurs. 2020 Mar;46(2):163-70.

19. Henderson C, Evans-Lacko S, Thornicroft G. Mental Illness Stigma, Help Seeking, and Public Health Programs. Am J Public Health. 2013 May;103(5):777-80.

20. Anderson ML, Dobkin C, Gross T. THE EFFECT OF HEALTH INSURANCE ON EMERGENCY DEPARTMENT VISITS: EVIDENCE FROM AN AGE-BASED ELIGIBILITY THRESHOLD. Rev Econ Stat. 2014;96(1):189-95.

21. Walsh M. The health belief model and use of accident and emergency services by the general public. J Adv Nurs. 1995;22(4):694-9. 
22. Leavitt F. The health belief model and utilization of ambulatory care services. Soc Sci Med Part Med Psychol Med Sociol. 1979 Jan 1;13:105-12.

23. Davis Cl, Montgomery AE, Dichter ME, Taylor LD, Blosnich JR. Social determinants and emergency department utilization: Findings from the Veterans Health Administration. Am J Emerg Med. 2020 Sep 1;38(9):1904-9.

24. Friedman B, Veazie PJ, Chapman BP, Manning WG, Duberstein PR. Is Personality Associated with Health Care Use by Older Adults? Milbank Q. 2013 Sep;91(3):491-527.

25. Chapman BP, Shah M, Friedman B, Drayer R, Duberstein PR, Lyness JM. Personality traits predict emergency department utilization over 3 years in older patients. Am J Geriatr Psychiatry Off J Am Assoc Geriatr Psychiatry. 2009 Jun;17(6):526-35.

26. Lutz BJ, Hall AG, Vanhille SB, Jones AL, Schumacher JR, Hendry P, et al. A Framework Illustrating Care-Seeking Among Older Adults in a Hospital Emergency Department. The Gerontologist. 2018 Sep 14;58(5):942-52.

27. He J, Hou X-Y, Toloo S, Patrick JR, Fitz Gerald G. Demand for hospital emergency departments: a conceptual understanding. World J Emerg Med. 2011;2(4):253-61.

28. Campbell Collaboration Systematic Reviews: Policies and Guidelines [Internet]. The Campbell Collaboration; 2014 May [cited 2021 Apr 12]. Available from: https://campbellcollaboration.org/library/campbell-collaboration-systematic-reviews-policies-andguidelines.html

29. Cochrane Handbook for Systematic Reviews of Interventions [Internet]. [cited 2021 Apr 12]. Available from: /handbook/current

30. Liberati A, Altman DG, Tetzlaff J, Mulrow C, Gøtzsche PC, loannidis JPA, et al. The PRISMA statement for reporting systematic reviews and meta-analyses of studies that evaluate healthcare interventions: explanation and elaboration. BMJ. $2009 \mathrm{Jul}$ 21;339:b2700.

31. Saran A. Training [Internet]. Campbell Collaboration. [cited 2021 Apr 12]. Available from: https://www.campbellcollaboration.org/research-resources/training-courses.html

32. Harrison JK, Reid J, Quinn TJ, Shenkin SD. Using quality assessment tools to critically appraise ageing research: a guide for clinicians. Age Ageing. 2017 May;46(3):359-65.

33. Ottawa Hospital Research Institute [Internet]. [cited 2021 Apr 12]. Available from:

http://www.ohri.ca/programs/clinical_epidemiology/oxford.asp

34. Herzog R, Álvarez-Pasquin MJ, Díaz C, Barrio JLD, Estrada JM, Gil Á. Are healthcare workers' intentions to vaccinate related to their knowledge, beliefs and attitudes? a systematic review. BMC Public Health. 2013 Dec;13(1):1-17.

35. WHO. Men, Ageing and Health. :63.

36. Barbabella F, Balducci F, Chiatti C, Cherubini A, Salvi F. Ecological factors associated with Emergency Department use by older people in Italy. Aging Clin Exp Res. 2021 Mar 1;33(3):659-68.

37. Hudson SM, Newman SD, Hester WH, Magwood GS, Mueller M, Laken MA. Factors influencing hospital admissions and emergency department visits among children with complex chronic conditions: a qualitative study of parents' and providers' perspectives. Issues Compr Pediatr Nurs. 2014 Mar 1;37(1):61-80.

38. Lowe RA, Fu R, Ong ET, McGinnis PB, Fagnan LJ, Vuckovic N, et al. Community Characteristics Affecting Emergency Department Use by Medicaid Enrollees. Med Care. 2009 Jan;47(1):15-22.

\section{Figures}




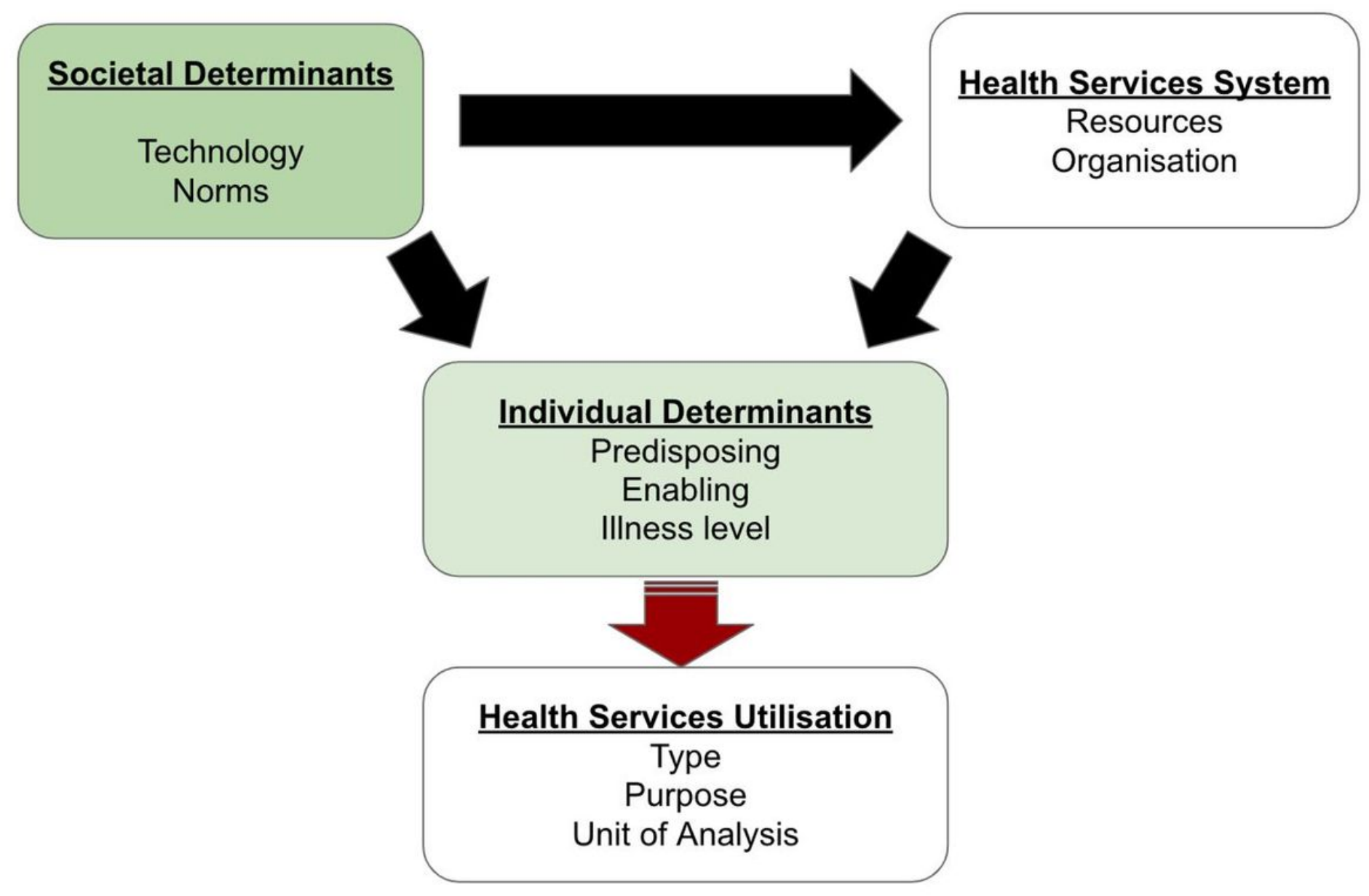

Figure 1

Framework for viewing Health Services Utilisation by Andersen and Newman 


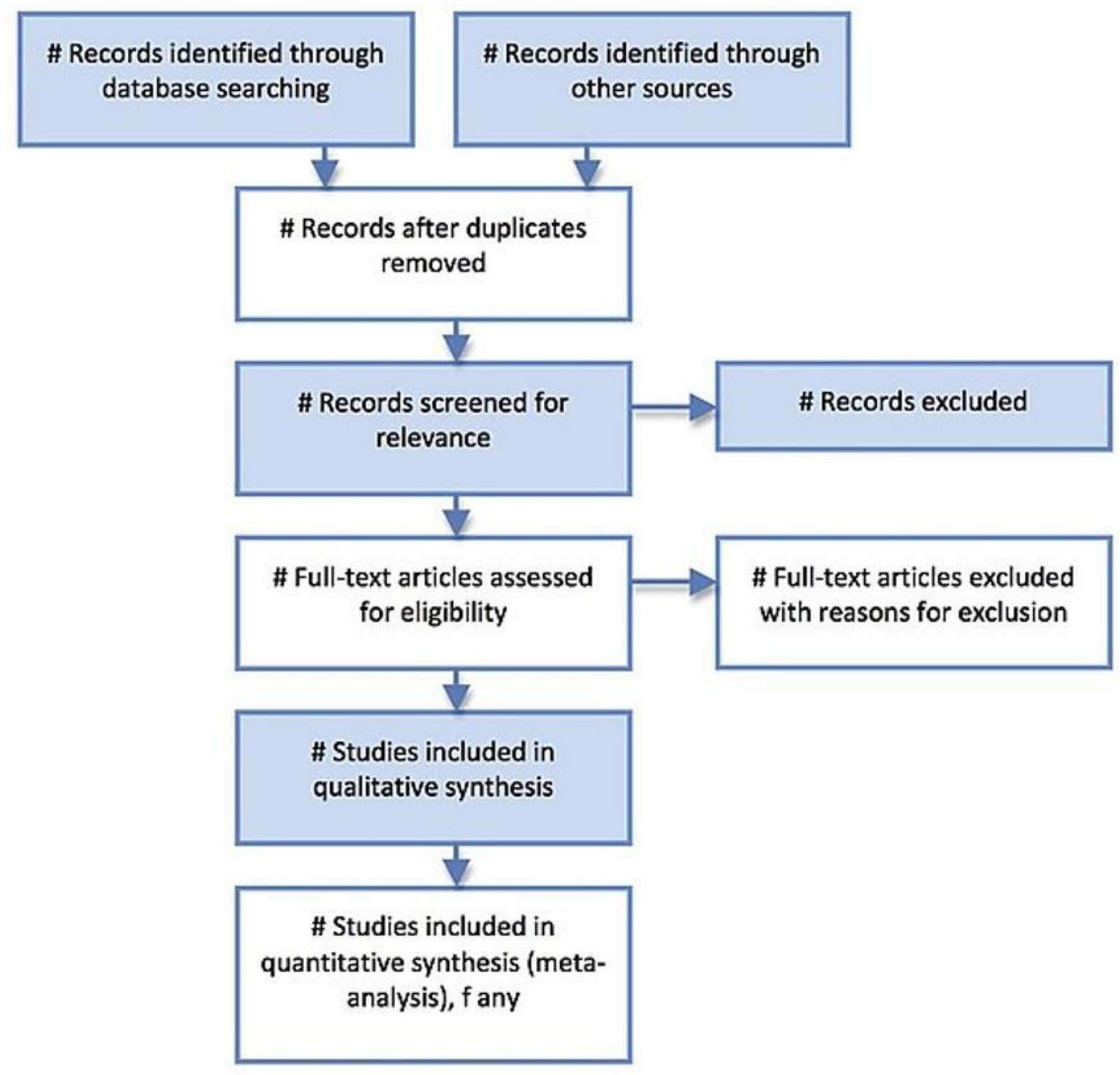

Figure 2

Sample PRISMA flow chart

\section{Supplementary Files}

This is a list of supplementary files associated with this preprint. Click to download.

- FullSearchStrategy.docx

- NOSforcrosssectional.pdf

- NOSscalecasecontrolandcohort.pdf

- PRISMAPchecklist.docx 